\title{
Three-octave-spanning supercontinuum generation and sub-two-cycle self-compression of mid-infrared filaments in dielectrics
}

\author{
Houkun Liang ${ }^{1}$, Peter Krogen ${ }^{1}$, Ross Grynko ${ }^{2}$, Ondrej Novak ${ }^{1}$, Chun-Lin Chang ${ }^{1}$, Gregory J. Stein ${ }^{1}$, \\ Darshana Weerawarne ${ }^{2}$, Bonggu Shim ${ }^{2}$, Franz X. Kärtner, ${ }^{1,3,4}$ and Kyung-Han Hong, \\ ${ }^{1}$ Department of Electrical Engineering and Computer Science and Research Laboratory of Electronics, \\ Massachusetts Institute of Technology (MIT), Cambridge, Massachusetts 02139, USA \\ ${ }^{2}$ Dept. of Physics, Applied Physics and Astronomy, Binghamton University, State University of New York, \\ Binghamton, New York 13902, USA \\ ${ }^{3}$ Center for Free-Electron Laser Science, DESY and Department of Physics, University of Hamburg, Hamburg, Germany \\ ${ }^{4}$ The Hamburg Center for Ultrafast Imaging, Luruper Chaussee 149, 22761 Hamburg, Germany \\ *Corresponding author: kyunghan@mit.edu \\ Received Month X, XXXX; revised Month X, XXXX; accepted Month X, XXXX; \\ posted Month X, XXXX (Doc. ID XXXXX); published Month X, XXXX

\begin{abstract}
We experimentally and numerically investigate the spectral and temporal structure of mid-infrared (mid-IR) filaments in bulk dielectrics with normal and anomalous group-velocity dispersion (GVD) pumped by a $2.1-\mu \mathrm{m}$ optical parametric chirped-pulse amplifier (OPCPA). Formation of stable and robust filaments with several $\mu \mathrm{J}$ of pulse energy is observed. We demonstrate more than 3-octave-spanning supercontinuum from $\mathrm{ZnS}$ in the normal GVD regime and selfcompression of the mid-IR pulse to sub-2-cycle duration in $\mathrm{CaF}_{2}$ in the anomalous GVD regime. The experimental observations quantitatively agree well with the numerical simulations based on a 3D nonlinear wave equation which reveals detailed spatio-temporal dynamics of mid-IR filaments in dielectrics.

OCIS codes: (320.6629) Supercontinuum generation, (320.7110) Ultrafast nonlinear optics, (320.5520) Pulse compression http://dx.doi.org/10.1364/OL.99.099999
\end{abstract}

Supercontinuum generation (SCG) has attracted extensive attention owing to its broad applications in sensing, spectroscopy, optical coherence tomography, and so on [1]. Extending SCG to the mid-IR range is highly desired for detecting biomedical materials and air pollutants with the resonant fingerprints of the common molecules, such as $\mathrm{H}_{2} \mathrm{O}, \mathrm{CO}_{2}, \mathrm{CO}$, and $\mathrm{NH}_{4}$. Moreover, coherent broadband mid-IR SC is a good candidate as the seed of mid-IR optical parametric amplifiers (OPAs) and OPCPAs for strong-field physics and attosecond science. In general, high-energy, few-cycle mid-IR pulses are extremely useful for the time-resolved studies of the fundamental processes in physics and chemistry.

The research work on SCG [2-5] and pulse compression [6] in the mid-IR range has been mostly explored in fibers in the past decade. SCG covering the whole mid-IR 'fingerprint region' has been demonstrated very recently in a chalcogenide step-index fiber pumped by $4.5 \mu \mathrm{m}$ and $6.3 \mu \mathrm{m}$ lasers [7]. However, there are intrinsic peak power limitations and alignment sensitivity issue with the method of fiber-based SCG. Recently, filamentation in bulk dielectrics started to show new opportunities with its advantages such as flexible materials available in normal and anomalous GVD regimes, no limitation of confinement loss at long wavelengths, high adaptable pump power, and high throughput. Multi-octave spanning SCG and the feasibility of few-cycle self-compression have been studied in a YAG crystal, pumped by femtosecond pulses at 3.1 $\mu \mathrm{m}[8,9]$.

In this Letter, we use a pump wavelength at $2.1 \mu \mathrm{m}$, generated from an OPCPA, for laser filamentation [10] in the Mid-IR regime. This wavelength is compatible to recently developed ultrafast laser amplifiers based on $\mathrm{Tm}^{-}$ or Ho-doped material [11]. We systematically investigate mid-IR SCG from filaments in different dielectrics in both normal and anomalous GVD regimes. More than 3-octave broadband spectrum is demonstrated for the first time in $\mathrm{ZnS}$ in the normal GVD regime. Moreover, mid-IR pulse self-compression down to sub-2 cycle duration is demonstrated in $\mathrm{CaF}_{2}$ in the anomalous GVD regime. Up to $9 \mu \mathrm{J}$ of pulse energy from the $\mathrm{SC}$ has been generated.

For a systematic study of the SCG in the mid-IR, $\mathrm{CaF}_{2}$ (5-mm thick), $\mathrm{BaF}_{2}$ (6-mm thick), and $\mathrm{ZnS}$ (8-mm thick), which have relatively flat dispersion around $2 \mu \mathrm{m}$ were chosen as bulk dielectric materials. The zero-dispersion wavelength (ZDW) of the three materials is $1.67 \mu \mathrm{m}, 1.92$ $\mu \mathrm{m}$, and $3.6 \mu \mathrm{m}$, respectively, as shown in Fig. 1(a). The bulk dielectrics were pumped by a $2.1 \mu \mathrm{m}, 34 \mathrm{fs}, 1 \mathrm{kHz}$ OPCPA with passive carrier-envelope phase stabilization [12]. The pump pulse energy was varied in the range from 0.1 to $11 \mu \mathrm{J}$ for optimization of the SCG process towards ultrabroad bandwidth and stable single filamentation. The pump beam was focused using an $f$ $=100 \mathrm{~mm} \mathrm{CaF} 2$ lens to a $1 / e^{2}$ diameter of $200 \mu \mathrm{m}$. We placed the input surface of dielectrics $4-7 \mathrm{~mm}$ before the focal spot to 1) enable the end of the filament to be just at the output surface of the dielectrics; and 2) avoid focusing at the dielectric surface to cause damage. Three different spectrometers based on $\mathrm{Si}$, InGaAs, and PbSe detectors, respectively, were employed to cover the spectra spanning from the visible to $\sim 5 \mu \mathrm{m}$ of wavelength. 

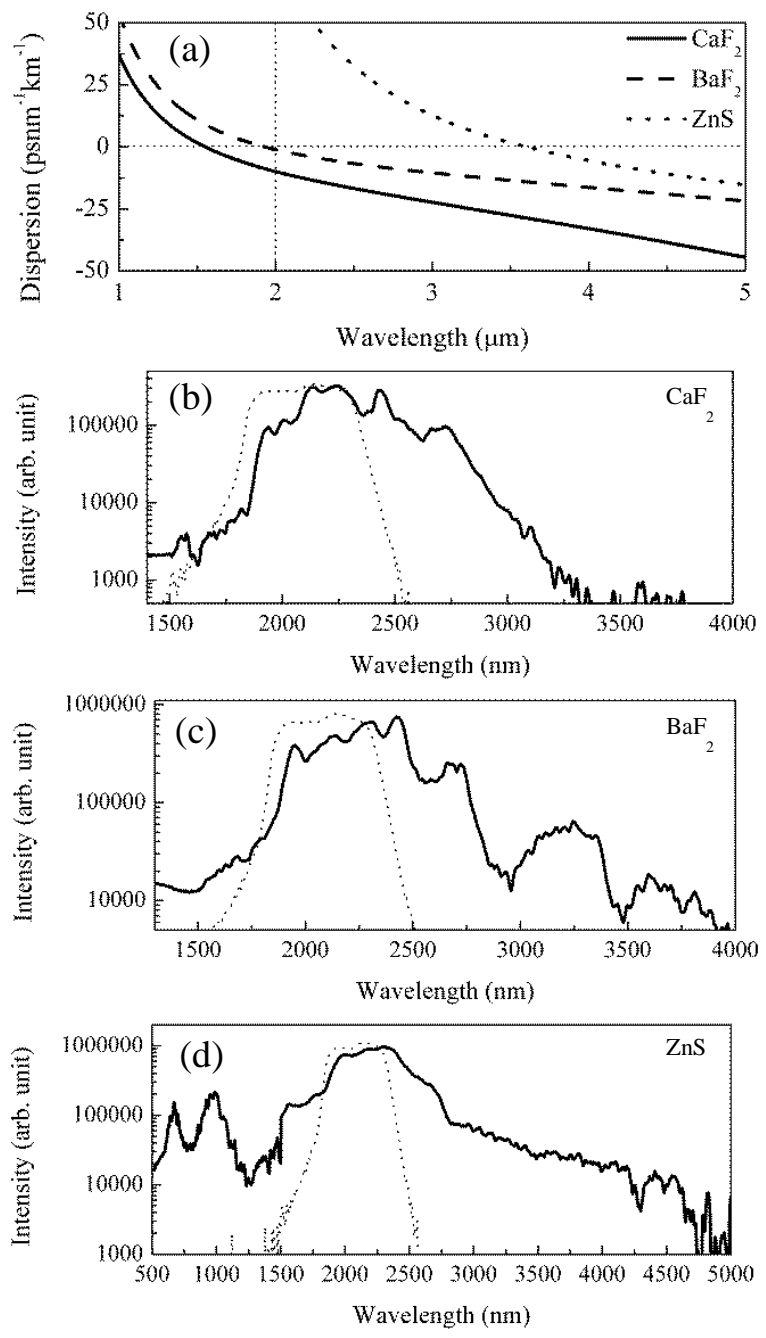

Fig. 1. (a) Material dispersion of $\mathrm{CaF}_{2}$ (solid), $\mathrm{BaF}_{2}$ (dash), and $\mathrm{ZnS}$ (dot). The generated SC from (b) 5-mm-thick $\mathrm{CaF}_{2}$ at $9 \mu \mathrm{J}$, (c) 6-mm-thick $\mathrm{BaF}_{2}$ at $7 \mu \mathrm{J}(\mathrm{c})$, and (d) 8-mm-thick $\mathrm{ZnS}$ at $5 \mu \mathrm{J}$. The dotted curves in (b), (c), and (d) are the spectra of the $2.1 \mu \mathrm{m}$ pump laser.

As shown in Figs. 1(b) and (c), with the pump wavelength at $2.1 \mu \mathrm{m}$, the continuum spectrum is extended to wavelengths up to $\sim 3.4 \mu \mathrm{m}$ and $\sim 4 \mu \mathrm{m}$ in $\mathrm{CaF}_{2}$ and $\mathrm{BaF}_{2}$, respectively. The deeper extension into the mid-IR in $\mathrm{BaF}_{2}$ is due to its flatter and lower dispersion, and thus broader conversion bandwidth with less temporal walk-off. Moreover, we performed SCG in a ZnS crystal with normal GVD, for the first time. As shown in Fig. 1(d), the generated SC spans from $500 \mathrm{~nm}$ to 4.5 $\mu \mathrm{m}$, corresponding to 3.1 octave. The peaks at $\sim 980 \mathrm{~nm}$ and $\sim 670 \mathrm{~nm}$ are observed, where the 670-nm component is possibly the third harmonic of the $2.1 \mu \mathrm{m}$ pump pulse. It is interesting that $\mathrm{ZnS}$ has a nonlinear refractive index $\left(n_{2}\right)$ as high as $90 \times 10^{-16} \mathrm{~cm}^{2} / \mathrm{W}$, which is $\sim 50$ times larger than that of $\mathrm{CaF}_{2}$. Surprisingly, we were still able to maintain a very stable filament with an input energy of $11 \mu \mathrm{J}$, which corresponds to 1000 times the critical power for self-focusing. The normal GVD seems to help the stable filamentation at over-critical power by rapidly broadening the pulse, but further investigation is needed to understand the detailed mechanism.

The visible images of the far-field pattern and the filaments inside $\mathrm{CaF}_{2}, \mathrm{BaF}_{2}$, and $\mathrm{ZnS}$ are captured. Stable conical far-field profiles were observed for $\mathrm{SC}$ from $\mathrm{CaF}_{2}$ and $\mathrm{BaF}_{2}$ as shown in Fig. 2(a) and (b), while $\mathrm{ZnS}$ has a more uniform far-field pattern shown in Fig. 2(c). The filaments from all the three dielectrics manifest a robust single filament as shown in Fig. 2 (d), (e), and (f). However, it is possible that multiple micro-filaments are generated in $\mathrm{ZnS}$ due to the highly over-critical power.
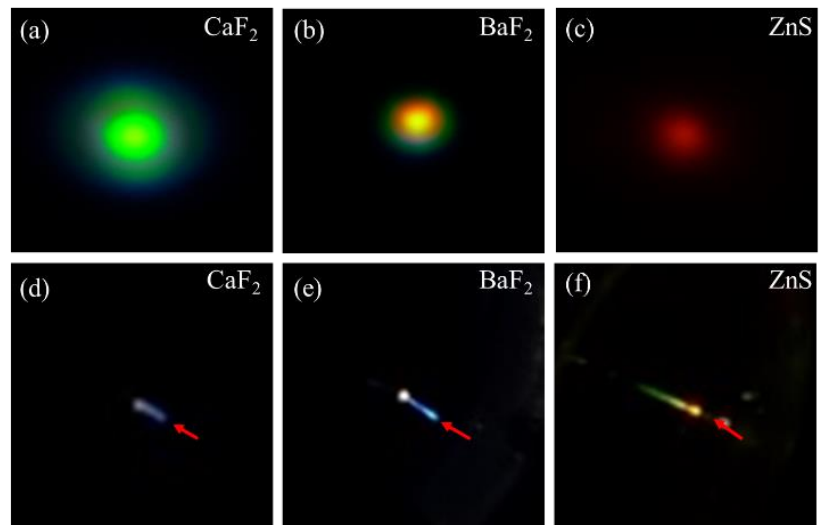

Fig. 2. The far field of the SC from (a) $\mathrm{CaF}_{2}$, (b) $\mathrm{BaF}_{2}$, and (c) $\mathrm{ZnS}$. (d), (e), and (f) are the visible images of their corresponding filaments inside the material, respectively. The red arrows indicate the incident direction of the pump pulses. (Color online)

The temporal profiles of the generated mid-IR SC were characterized using a second-harmonic generation (SHG) interferometric autocorrelator. A $100-\mu \mathrm{m}$ thick $b$-Barium Borate and a 400- $\mu \mathrm{m}$ thick $\mathrm{AgGaS}_{2}$ is used for the SHG of the $2.1 \mu \mathrm{m}$ pump pulse and the mid-IR filaments, respectively. A $900 \mathrm{~nm}$ long-pass filter and a $1500 \mathrm{~nm}$ short-pass filter were employed after SHG to ensure background free measurements, so that the mid-IR SC from 1800 to $3000 \mathrm{~nm}$ was characterized. As shown in Figs. 3(a) and $3(\mathrm{~b})$, with the $\sim 34 \mathrm{fs}$ pump pulse in full width at half maximum (FWHM), the $\mathrm{SC}$ from $\mathrm{BaF}_{2}$ was broadened to $\sim 49 \mathrm{fs}$ in FWHM. This is due to the self-phase-modulation (SPM) induced chirp that has not been well compensated by the weak anomalous GVD. In contrast, as shown in Fig. $3(\mathrm{c})$, from $\mathrm{CaF}_{2}$, which has stronger anomalous GVD, the generated SC was self-compressed to $<14 \mathrm{fs}$ in FWHM, corresponding to sub-two optical cycles, close to the transform-limited pulse duration for $1800-3000 \mathrm{~nm}$ of measurement window. This implies the SPM and strong anomalous GVD are almost perfectly balanced. The interferometric autocorrelation trace of $\mathrm{SC}$ in $\mathrm{CaF}_{2}$ shown in the inset of Fig. 3(c) was calculated from the 1800-3000 $\mathrm{nm}$ components of the measured SC spectrum with a flatphase assumption. The calculated autocorrelation trace matches well with the measurement, except there are some side lobes in the measured trace which is attributed to the high-order dispersion and temporal splitting. On the other hand, the measured duration of pulses from $\mathrm{ZnS}$ filaments are longer than 200 fs because the SC forms the dispersive wave. 

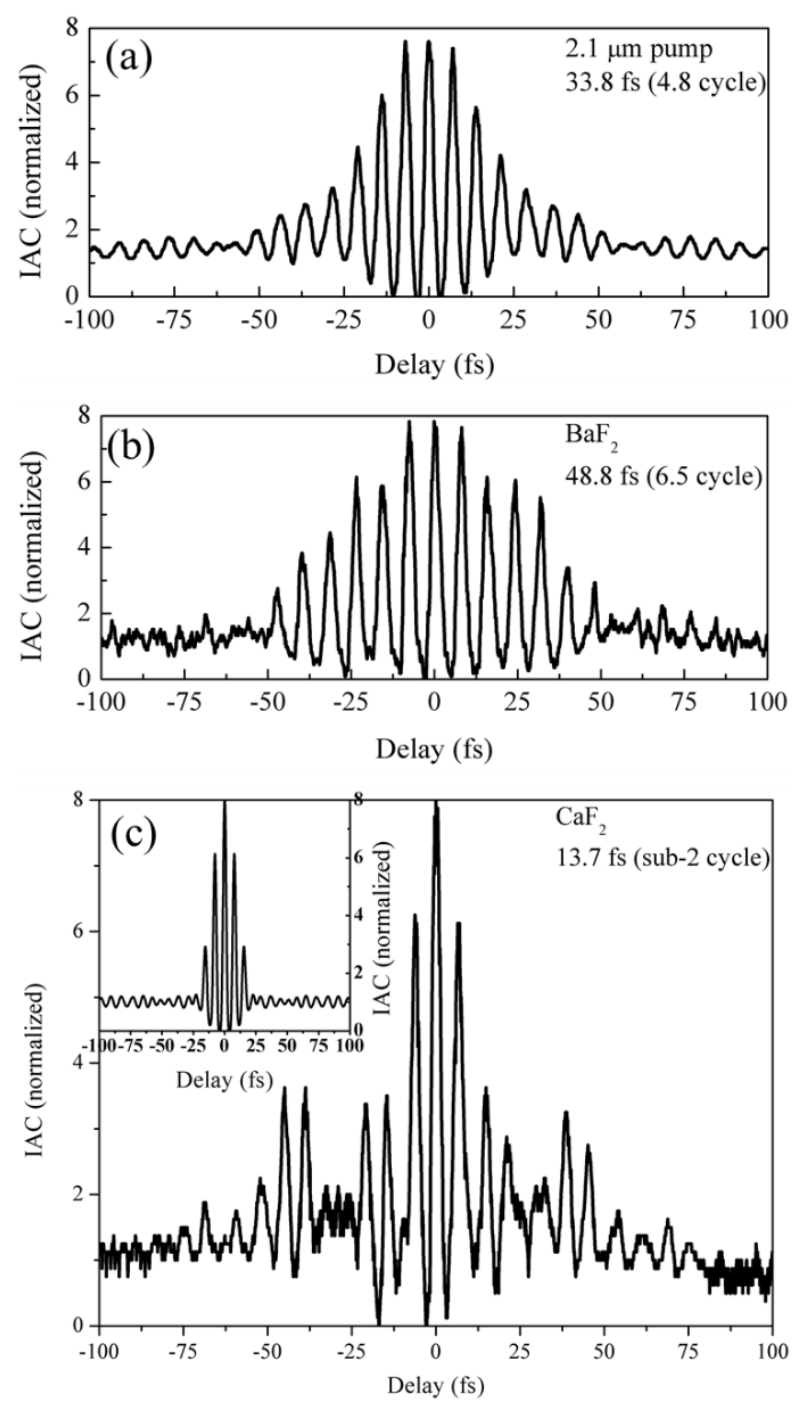

Fig. 3. The measured interferometric autocorrelation traces of the SC generated in (a) $2.1 \mu \mathrm{m}$ pump pulse, (b) $\mathrm{BaF}_{2}$, and (c) $\mathrm{CaF}_{2}$. The pulse energy of $6 \mu \mathrm{J}$ was used for both $\mathrm{CaF}_{2}$ and $\mathrm{BaF}_{2}$. The inset of (c) is the calculated autocorrelation trace from the 1800$3000 \mathrm{~nm}$ components of the measured spectrum.

To have more quantitative understanding about the experiments, we carried out numerical simulations using the nonlinear envelope equation (NEE) in normalized units which is given by $[13,14]$

$$
\begin{gathered}
\frac{\partial A}{\partial \xi}=\frac{i}{4}\left(1+\frac{i \partial}{\omega \tau_{p} \partial \tau}\right)^{-1} \nabla^{2} A+i L_{d f} \sum_{n=2}^{6} \frac{\beta_{n}}{n !}\left(\frac{i \partial}{\tau_{p} \partial \tau}\right)^{n} A \\
+i\left(1+\frac{i \partial}{\omega \tau_{p} \partial \tau}\right) \frac{L_{d f}}{L_{n l}}|A|^{2} A-\frac{L_{d f}}{2 L_{m p}} \frac{A}{|A|^{2}} \frac{\partial \eta}{\partial \tau} \\
-\frac{L_{d f}}{L_{p l}}\left(i+\frac{1}{\omega \tau_{c}}\right) \eta\left(A+\frac{\frac{\tau_{c} \partial A}{\tau_{p} \partial \tau}}{1-i \omega \tau_{p}}\right),
\end{gathered}
$$

where $A$ is the electric field normalized by the input electric field amplitude, $\xi$ is the propagation distance normalized by the input beam diffraction length $L d f, \omega$ is the central frequency of the laser pulse, $\tau$ is the retarded time normalized by the pulse duation $\tau_{p}, \omega$ is the angular frequency of the central wavelength, $B_{n}$ is the dispersion parameter (e.g., $b_{2}$ is GVD), $L_{n l}$ is the nonlinear length, $L_{m p}$ is the multi-photon absorption length, $L_{p l}$ is the plasma length, $\eta$ is the normalized electron density, and $\tau_{c}$ is the collision time. Each term on the right side of the equation represents diffraction, dispersion, self-focusing via Kerr-nonlinearity, multi-photon absorption, and plasma terms (defocusing and collisional absorption), respectively. Multi-photon ionization and avalanche ionization generate free electrons, which is described by

$$
\frac{\partial \eta}{\partial \tau}=|A|^{2 m}+\alpha \eta|A|^{2},
$$

where $m$ is the number of photons to ionize the medium, and $\alpha$ is the avalanche ionization coefficient.

The simulated SCG reproduces the experiment measurement with high accuracy as shown in Figs. 4(a) and (b). For $\mathrm{BaF}_{2}$, its calculated output spectrum has larger spectral components at 3-4 $\mu \mathrm{m}$ than that of $\mathrm{CaF}_{2}$, which is consistent with the experimental data. For $\mathrm{ZnS}$, the calculation also quantitatively reproduces the measured spectrum that there is significant spectral extension up to $>4.5 \mu \mathrm{m}$. Besides the $\mathrm{SC}$ spectra, temporal dynamics of the SCG in different dielectrics were also revealed by simulations. Pulses in the anomalous GVD regime i.e., in $\mathrm{BaF}_{2}$ and $\mathrm{CaF}_{2}$, shown in Figs. 4(c) and (d) are strongly localized near $t=0$ even though there is short-time scale pulse splitting due to plasma generated by the high intensity pulses. To the contrary, pulse broadening and pulse splitting are clearly observed in the normal GVD regime in ZnS [15], shown in Fig. 4(e). The spatially-averaged autocorrelation traces over entire spectral bandwidth were simulated for comparison with the measurement results. For the $\mathrm{SC}$ in $\mathrm{CaF}_{2}$, the autocorrelation trace in Fig. 4(f) shows a $17 \mathrm{fs}$ in FWHM, corresponding to a $12 \mathrm{fs}$ pulse width. This tallies well with the measured self-compressed sub-two-cycle pulse from the $\mathrm{SC}$ in $\mathrm{CaF}_{2}$ except that the measured pulse duration is longer because of the limited bandwidth. The imperfect self-compression in $\mathrm{BaF}_{2}$ is also well reproduced by simulations shown in Fig. 4(g). On the other hand, ZnS broadens the autocorrelation to $\sim 300$ fs due to the normal GVD and pulse splitting, which is also consistent with the measurement.

In conclusion, we systematically investigated the midIR SCG from filaments in bulk dielectrics with normal and anomalous GVD. 3-octave-spanning SC spectrum in $\mathrm{ZnS}$ with normal GVD was generated with robust and stable output power. In the anomalous GVD regime, subtwo-cycle self-compression of mid-IR filaments from $\mathrm{CaF}_{2}$ was experimentally observed. Filaments have several $\mu \mathrm{J}$ of pulse energy. Such a coherent broadband source with few-cycle duration in the mid-IR range is a promising seed source for seeding mid-IR OPA and OPCPA as well as a good candidate for time-resolved tools for molecular motion study. 

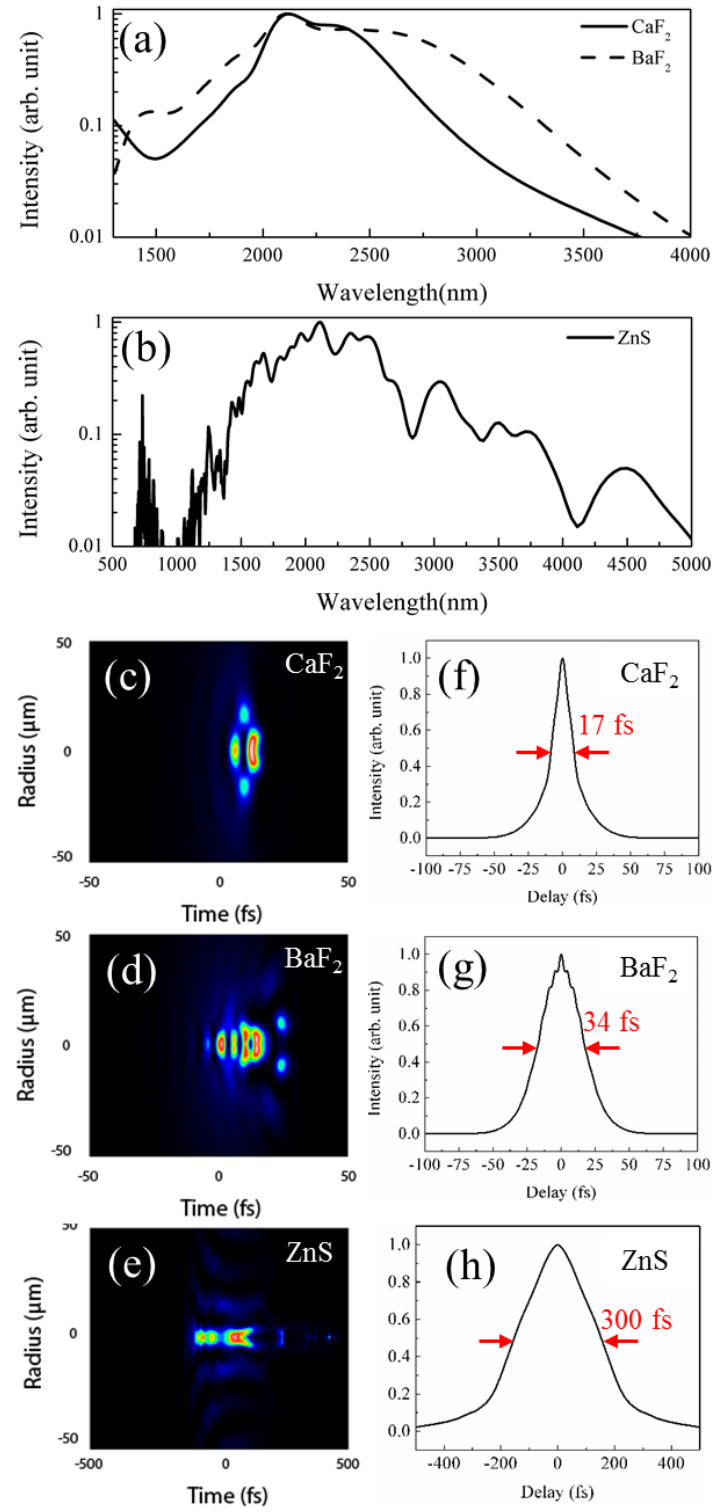

Fig. 4. Simulated spatially-averaged spectra in (a) $\mathrm{CaF}_{2}$ and $\mathrm{BaF}_{2}$, and (b) ZnS. Simulated spatio-temporal profiles when $2.1 \mu \mathrm{m}, 30$ fs pulses pass through (c) $\mathrm{CaF}_{2}$, (d) $\mathrm{BaF}_{2}$, and (e) $\mathrm{ZnS}$. Ten-time larger time scale is used in (e). Simulated spatially-averaged autocorrelation traces of $\mathrm{SC}$ in (f) $\mathrm{CaF}_{2},(\mathrm{~g}) \mathrm{BaF}_{2}$, and (h) $\mathrm{ZnS}$. The pulse energies used for simulation are $6 \mu \mathrm{J}$ for $\mathrm{CaF}_{2}$ and $\mathrm{BaF}_{2}$, and $5 \mu \mathrm{J}$ for $\mathrm{ZnS}$.

The authors would like to thank Dr. Jeffrey Moses for providing the mid-IR spectrometer and autocorrelator. This work was supported by AFOSR (FA9550-12-1-0499, FA9550-12-1-0080, FA9550-13-1-0159, and FA9550-14-10255), the Center for Free-Electron Laser Science, DESY, Hamburg, Germany, and the excellence cluster "The Hamburg Centre for Ultrafast Imaging-Structure, Dynamics and Control of Matter at the Atomic Scale" of the Deutsche Forschungsgemeinschaft. H. K. Liang acknowledges the financial support from Singapore Institute of Manufacturing Technology (SIMT/14-110023) and Agency for Science, Technology and Research
(A*STAR), Singapore. R. Grynko, D. Weerawarne, and B. Shim acknowledge the financial support from SUNY Research Foundation. O. Novak acknowledges the European Regional Development Fund, the European Social Fund and the state budget of the Czech Republic (project HiLASE: CZ.1.05/2.1.00/01.0027, DPSSLasers: CZ.1.07/2.3.00/ 20.0143, Postdok: CZ.1.07/2.3.00/30.0057). His current affiliation is HiLASE Centre, Institute of Physics ASCR, Czech Republic. C.-L. Chang acknowledges the Ministry of Science and Technology in Taiwan for the Postdoctoral Research Abroad Program NSC 102-2917-I-564-026.

\section{References}

1. R. R. Alfano, 'The Supercontinuum Laser Source', Springer-Verlag, New York, (1989);

2. P. Domachuk, N. A. Wolchover, M. Cronin-Golomb, A. Wang, A. K. George, C. M. B. Cordeiro, J. C. Knight, F. G. Omenetto, Opt. Express 16, 7161 (2008);

3. G. Qin, X. Yan, C. Kito, M. Liao, C. Chaudhari, T. Suzuki, Y. Ohishi, Appl. Phys. Lett. 95, 161103 (2009).

4. C. Xia, M. Kumar, O. P. Kulkarni, M. N. Islam, J. F. L. Terry, M. J. Freeman, M. Poulain, G. Mazé, Opt. Lett. 31, 2553 (2006);

5. W. Yang, B. Zhang, G. Xue, K. Yin, J. Hou, Opt. Lett. 39, 1849 (2014);

6. A. A. Amorim, M. V. Tognetti, P. Oliveira, J. L. Silva, L. M. Bernardo, F. X. Kärtner, H. M. Crespo, Opt. Lett. 34, 3851 (2009);

7. C. R. Petersen, U. Møller, I. Kubat, B. Zhou, S. Dupont, J. Ramsay, T. Benson, S. Sujecki, N. AbdelMoneim, Z. Tang, D. Furniss, A. Seddon, O. Bang, Nat. Photon. 8, 830 (2014);

8. F. Silva, D. R. Austin, A. Thai, M. Baudisch, M. Hemmer, D. Faccio, A. Couairon, J. Biegert, Nat. Commun. 3, 807 (2012);

9. M. Hemmer, M. Baudisch, A. Thai, A. Couairon, J. Biegert, Opt. Express 21, 28095 (2013);

10. For example, A. Couairon and A. Mysyrowicz, Phys. Rep. 441, 47 (2007);

11. A. Dergachev, Proc. SPIE 8599, Solid State Lasers XXII: Technology and Devices, 85990B (18 March 2013); doi: 10.1117/12.2001386

12. J. Moses, S.-W. Huang, K.-H. Hong, O. D. Mücke, E. L. Falcão-Filho, A. Benedick, F. Ö. Ilday, A. Dergachev, J. A. Bolger, B. J. Eggleton, F. X. Kärtner, Opt. Lett. 34, 1639 (2009);

13. A. L. Gaeta, Phys. Rev. Lett. 84, 3582 (2000);

14. B. Shim, S. E. Schrauth, A. L. Gaeta, Opt. Express 19, 9118 (2011);

15. J. K. Ranka, R. W. Schirmer, A. L. Gaeta, Phys. Rev. Lett. 77, 3783 (1996). 